\title{
Matching of Iris Pattern Using Image Processing
}

\author{
N.B. Prajwala, N.B. Pushpa
}

\begin{abstract}
Personal identification is very vital in this digital era for simpler mobile phone unlocking to criminal identification in the scene of crime. There are various methods of personal identification ranging from non-invasive methods of presence of moles in the visible parts of the body to the invasive DNA karyotyping. Other in the spectrum being fingerprinting, lip print, foot print, tongue print, palate print etc. As age advances there might be slight variations in finger print, ear biometric etc, where as in iris the amount of pigmentation might vary but the pattern remains almost same from birth to death, unless otherwise there is any injury to the iris which is very remote. Iris pattern recognition is a non-invasive method of biometric identification. Iris architecture is not only complex but also unique to an individual. In this article a methodology is been proposed to match iris pattern.
\end{abstract}

Keywords--- Canny Edge Detection, Correlation Co-efficient, Iris Biometric, Iris Pattern Matching.

Iris recognition by pattern matching is the process of identifying a person by analyzing the pattern of the iris. Iris is a thin, shallow, cone shaped structure surrounding the pupillary aperture with root located posteriorly compared to free (pupillary) margin. From person to person the iris size varies with a range of 10.2 to $13.0 \mathrm{~mm}$ and an average size of $12 \mathrm{~mm}$ diameter and a circumference of $37 \mathrm{~mm}$. Although iris is named after the rainbow its range of colours includes only from blue to darkest brown, which varies in the two eyes of an individual and even within the same iris. Colour of the iris is due to the amount of pigment cells which selectively absorbs and reflects the light of different frequencies. The size of pupil present in the centre of iris dilates when light falls on it and contacts on decrease in the intensity of light. For this research the light intensity is maintained constant while capturing iris image to get proper pattern of iris.

Digital image processing is used as a tool for iris pattern matching. Image processing has a wide range of inbuilt algorithms and filters for processing image. The iris pattern of ridges and folds (formed by vessels and nerves supported by connective tissue) varies from person to person. For the ID purpose the unique iris character can be used. Iris patterns are much more unique compare to fingerprints. Error rate of iris is less than one in million compared to variation in face and finger print of a person as he gets older whereas the iris pattern is considered to remain constant. Many attempts like edge detection, pattern matching are been made for iris biometric. Here one such attempt is made; filters like RGB to grey, canny edge detection, and

\footnotetext{
Manuscript received September 16, 2019.

N.B. Prajwala, Department of Studies in Computer Science, University of Mysore, Mysore. (e-mail: prajwala.nb4@gmail.com)

Dr.N.B. Pushpa, Department of Anatomy, JSS Medical College

JSSAHER, Mysore. (e-mail: pushpanb@jssuni.edu.in)
}

\section{INTRODUCTION} finger print and facial recognition. There are more chance of

morphological operations are performed on the image to get better accurate results.

\section{RELATED WORK}

The methodology canny edge detection used in [1] work gave good results. This method successfully identifies and also matches the iris from the database. The system need to be trained before recognizing the iris. The use of database has made the system more usable and compatible. Iris is a circle with an average diameter of $12 \mathrm{~mm}$ and circumference of $37 \mathrm{~mm}$.

The work in [2] can be used to detect the circle at the starting phase and then to proceed with pattern matching. The author has given clear and efficient methods to find circle. [3] In this the author has elaborated several efficient techniques for iris recognition. It gives clear idea about what is existing systems and what can be done or enhanced. The article gives clear picture about iris structure and iris anatomy has been elaborated in detail. He has also illustrated the limitations and advantages of existing systems.

\section{RESEARCH METHODOLOGY \& RESULTS}

This system has series of sub system which correspond to every step of iris pattern matching. The stages are:

- Image acquisition.

- RGB to gray scale image.

- Canny edge detection.

- Area opening.

- Imclose morphological operation.

- Imfill

- Bwboundaries.

- Correlation coefficient.

\section{Image acquisition}

Image acquisition is a process of capturing image. This is very important stage for this system since the pattern and structure of iris has to be clear.

The pupil present in the centre of iris has a property of enlarging and contracting depending on the intensity of light falls on it, hence the image should be taken under fixed light intensity.

The photographs of iris images are taken using slit-lamp microscope; it is a low powered microscope which has a slit lamp with high intensity light.

This slit lamp has different filters to get different views of the eyes. After image acquisition image is resized to desired dimension and size. 

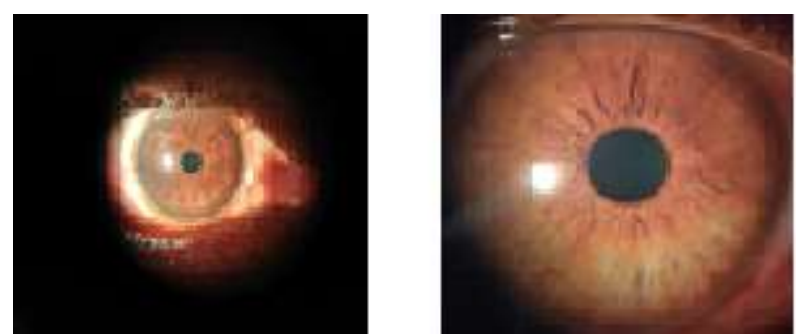

Fig. 1: Image acquisition and resizing

\section{2. $R G B$ to gray scale image}

rgb2gray filter is applied on image. This filter gives gray scale intensity image by eliminating hue saturation and retains luminance. It eliminates all colours in the image and gives a black and white image or gray scale image as shown in figure 2
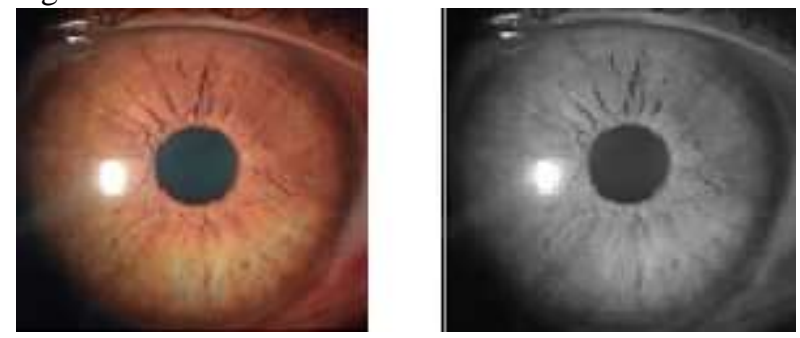

Fig. 2: Image conversion from RGB to gray scale

\section{Canny edge detection}

It is a process of finding boundaries in the images. Discontinuities in brightness are detected. Edge detection are used for data extraction and segmentation. In this canny edge filter is applied for the gray scaled image to get the clear patterns present in the iris.
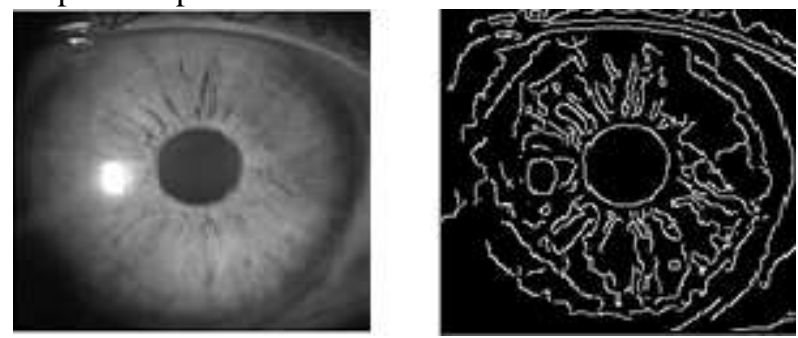

Fig. 3: Canny edge image of iris

\section{Area opening and imclose morphological operation}

Area opening function is used on the edge detected image. It is a function to remove connected components smaller than specified pixels from the binary image and it gives a new binary image. A structuring element of disk size 2 is created; structuring elements are used for morphological operations. Imclose morphological operation is performed on the binary image that is dilation followed by erosion. Dilation is a process of getting maximum value of all pixels in the input neighbourhood pixel's whereas erosion is a process of getting minimum value of all pixels in the input neighbourhood pixel's.
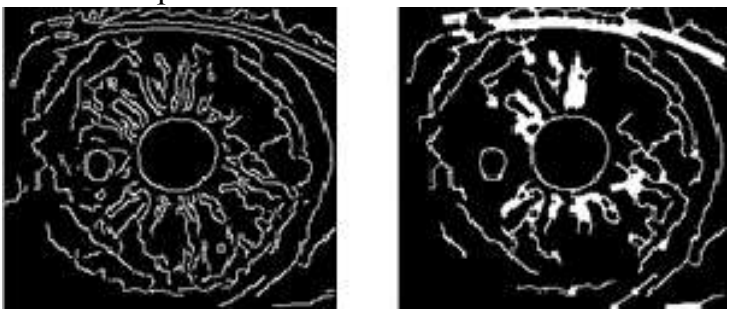

Fig. 4: Morphological operation on iris image

\section{Imfill and bwboundaries}

Imfill and bwboundries are applied to get clear structure and pattern of iris and pupil. This performs flood-fill operation on background pixels of the input binary image. Bwboundaries are used to extract the boundaries of exterior objects and inside hole boundaries present in the binary images.
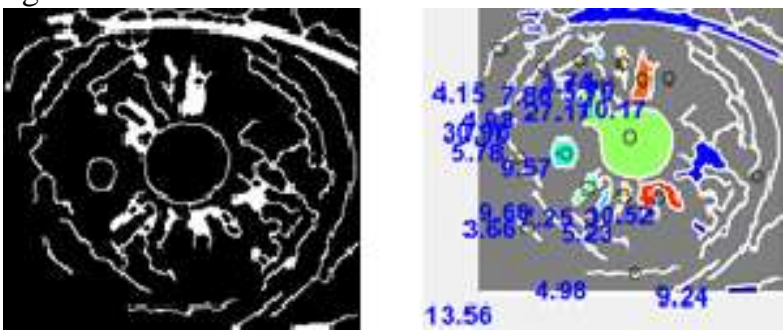

Fig. 5: Imfill and bwboundaries on iris image

\section{Correlation coefficient}

Iris images to be compared are applied with above filters and then correlation coefficient between processed images is determined. The correlation coefficient returns the numerical difference value between two iris images. If the correlation coefficient is 1 then the iris matches that is the iris images taken belongs to same person if the value varies then the iris belongs to different persons. If the iris pattern matches the resultant images will be as shown in the figure8. If the iris pattern doesn't match then the resultant image will be as shown in figure9, the difference in the colour shows the pattern difference between two iris.

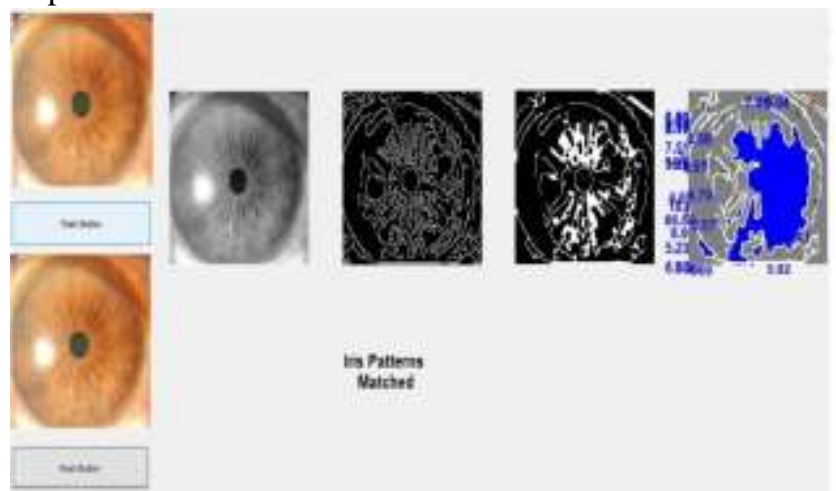

Fig. 6: Matched iris patterns
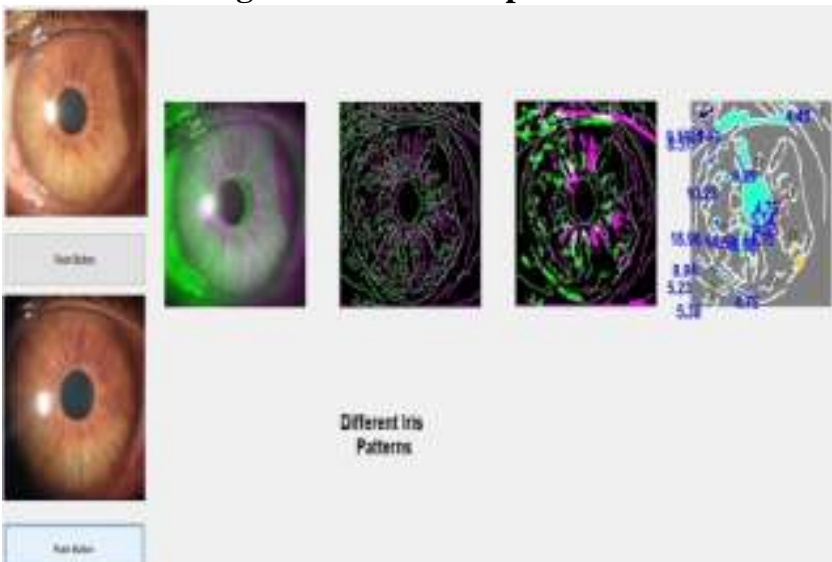

Dffeient ins

son

Fig. 7: Different iris patterns

Published By:

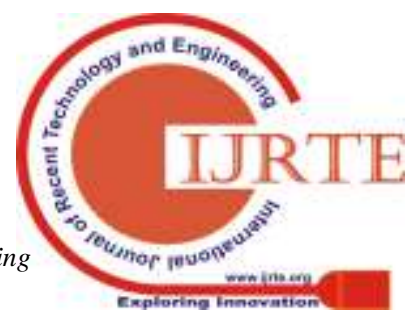


This methodology works well even when the iris colours are different as shown in figures 8 and 9.

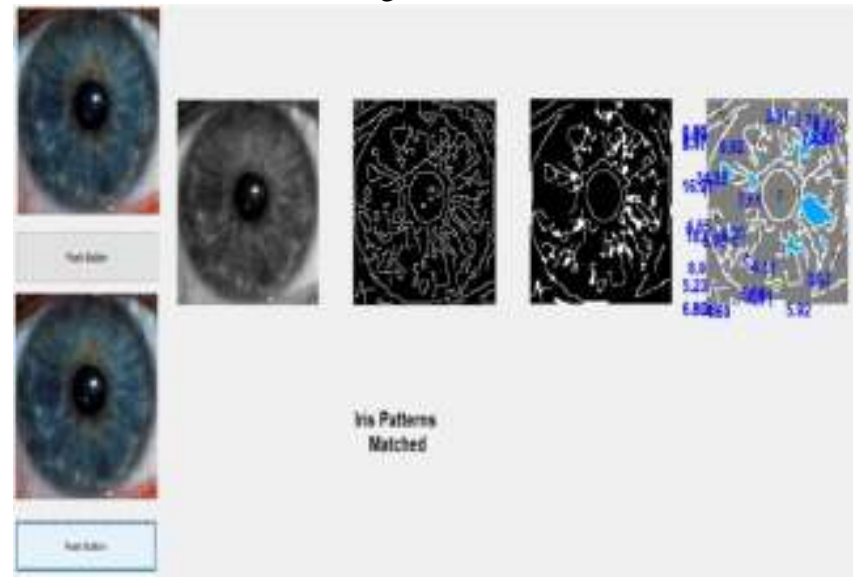

Fig. 8: Pattern matched for different coloured iris

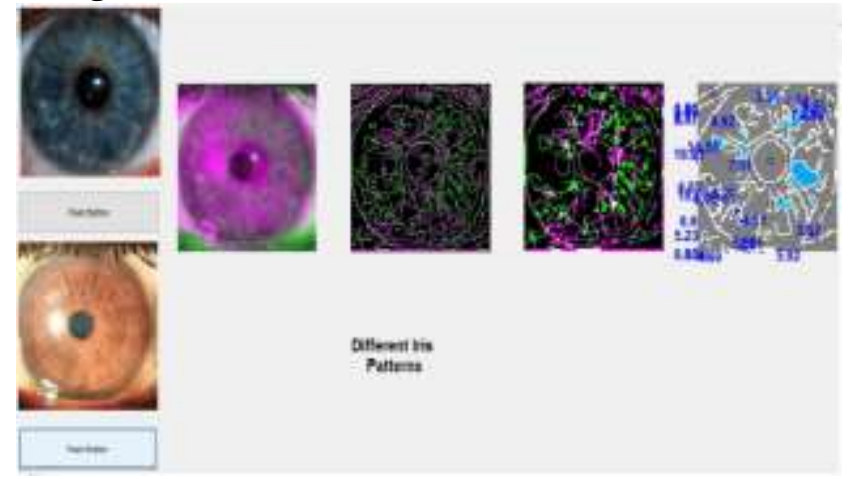

Fig. 9: Different pattern for different coloured iris

\section{CONCLUSION}

Iris recognition is an automated pattern matching technique for biometric identification. The complex iris patterns which are unique even in the right and left eye of the same individual. The results from the study are comparatively accurate. But the results of matching can vary even if there is slight change in the intensity of light thrown on the iris while capturing. The limitation is that capturing of clear accurate iris image to show the detailed rich patterns and colour of the iris is challenging. Images used for this work are taken from slit-lamp microscope, which are commonly used by Ophthalmologist. This work can be progressed to detect left and right iris and further more for authentication, person identification in medico legal cases.

\section{REFERENCES}

1. Neha Kak, Rishi Gupta, Sanchit Mahajan. Iris Recognition System, (IJACSA) International Journal of Advanced Computer Science and Applications, Vol. 1, Vol. 1, 2010 Page | 34.

2. T. Chuan Chen, K. Liang Chung: An Efficient Randomized Algorithm for Detecting Circles. Computer Vision and Image Understanding Vol. 83 (2001) 172-191.

3. A. Alice Nithya, A. Alice Nithya. Iris Recognition Techniques: A literature Survey, International Journal of Applied Engineering Research · July 2015.

4. A. K. Jain, A. Ross, and S. Prabhakar, "An Introduction to Biometric Recognition," IEEE
Trans. Circuits Syst. Video Technol., vol. 14, pp. 4-20, 2004.

5. http://en.wikipedia.org/wiki/Iris_recognition (accessed on Jun, 2014).

6. Mark Hill, “ANAT2310: Eye Development," The University of South Wales, 2003.

7. Barbara Westmoreland, Michael Lemp, and Richard Snell, Clinical Anatomy of the eye 2nd edition, (Oxford :Blackwell Science Inc., 1998).

8. Clyde Oyster, The Human Eye Structure and Function, Sinauer Associates, 1999.

9. Bertillon. La couleur de Piris. Revue scientifique, Vol. 36, No. 3, pp. 65-73, 1885.

10. Leonard Flom and AranSafir, "Iris recognition system", U.S.Patent 1244552, 1987

11. N B, Prajwala. Defect Detection in Pharma Pills Using Image Processing. International Journal of

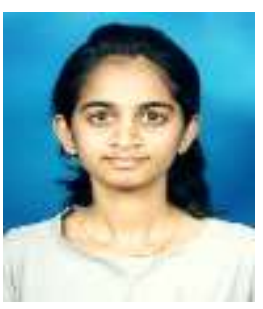
Engineering \& Technology, [S.1.], v. 7, n. 3.3 , p. $102-106$, june 2018. ISSN 2227-524X.

Prajwala, N.B, Mysuru, Karnataka. MTech in Computer Science in the year 2016 from University of Mysuru. Bachelor of Engineering in Computer Science in the year 2013. Was selected as an intern to L\&T, Mysuru during 2015. Worked as an intern in Aplus intellitech solution for one year on embedded $\mathrm{c}$ and on image processing. Worked as a team lead in a plus intellitech solution. Worked as software developer in Inqola. Also worked as quality analyst. Was faculty cum research associate in the department of computer science, Amrita School of arts and science, Mysuru, Amrita Vishwa Vidyapeetham, mysore, Imdia. Have published three research articles on image processing which are indexed in scopus, "Defect Detection in Pharma Pills Using Image Processing" in International Journal of Engineering \& Technology during 2018, "Identification of Fake Notes and Denomination

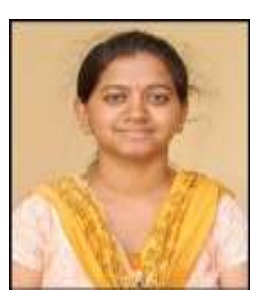
Recognition" and "Facial Expression Recognition by Calculating Euclidian Distance for Eigen Faces Using PCA" in IEEE explorer.

Dr. Pushpa, N.B, Mysuru, Karnataka. MD in Anatomy in the year 2014 from RGUHS, Karnataka. Completed MBBS in the year 2009. Currently working has Assistant Professor, department of Anatomy, JSS Medical college, JSSAHER, Mysore. Have published six articles in national and international journals. Sexual dimorphism of Lip prints in south Indian Population in Indian journal of clinical Anatomy and Physiology is one among them. Participated in various CMEs, State and National conferences. Is a life member of Karnataka Chapter of Anatomists, Anatomical society of India and Society of clinical Anatomists. Have won best poster award at national conference to combat depression. Areas of interest are genetics and medical education. 